\title{
Bayesian modelling of gene network alterations during blood cells differentiation and cancerogenesis
}

\author{
A.A. Igolkina*, M.G. Samsonova \\ Peter the Great St. Petersburg Polytechnic University, Mathematical Biology and Bioinformatics \\ Laboratory, St. Petersburg, Russia \\ *e-mail: igolkinaanna11@gmail.com
}

Key words: gene network, Structural Equation Modeling, Bayesian inference, branching process, Wiener process, Leukemia

Blood is a heterogeneous tissue with the known tree of differentiation. While the process of differentiation from hematopoietic stem cells through different progenitor states to mature states (monocytes, lymphocytes, neutrophils, etc.) is continuous we hypothesise that changes in genetic networks along the tree also follow the continuous-states process. We developed the model of one gene network alterations along the tree based on the following assumptions. (1) In each inner node and outer leaf of the tree, gene network satisfies the Structural Equation Model (SEM); (2) the change of gene covariance matrix together with coefficients of gene-gene interactions follow the continuous-states, timehomogeneous Markov Process, specifically the Wiener Process. We used gene expression data within the leaves (microarray Human Map dataset) and optimised all parameters of both SEM model and the Wiener Process drawing the Bayesian Inference. We worked with RAS signalling network as it involves in Leukemia development. We predicted the states of this gene network in inner nodes and, using parameters of the Wiener Process, predicted the point on the tree where the cancer cells (T-cells and B-cells) have its own branch. The knowledge of this point can potentially help in leukaemia treatment. We consider, the developed methodology can be easily applied to other cell development and also phylogenetic studies. 